\title{
Satisfacción de residentes quirúrgicos con la reestructuración del modelo de enseñanza durante la pandemia de COVID-19
}

\section{Surgical resident satisfaction with educational restructuring during the COVID-19 pandemic}

\author{
Jorge Luis Martínez-Peniche, ${ }^{*}$ Francisco Guillermo Castillo-Vázquez, ${ }^{*}$ Carlo Enrico Bañuelos-Aluzzi, ${ }^{*}$ \\ José Enrique Villegas-del Ángel, ${ }^{*}$ Roberto Pablo Pareyón-Valero*
}

Citar como: Martínez-Peniche JL, Castillo-Vázquez FG, Bañuelos-Aluzzi CE, Villegas-del Ángel JE, Pareyón-Valero RP. Satisfacción de residentes quirúrgicos con la reestructuración del modelo de enseñanza durante la pandemia de COVID-19. An Med ABC. 2021; 66 (2): 91-96. https://dx.doi.org/10.35366/100476

\section{RESUMEN}

Introducción: La pandemia de COVID-19 obligó a la rápida reestructuración de los recursos humanos en salud. Este cambio provocó que la educación médica se viera afectada, dando prioridad a la atención médica de pacientes infectados por COVID-19. Objetivo: Medir la satisfacción de residentes quirúrgicos del Centro Médico $\mathrm{ABC}$ con el cambio en el modelo educativo y exponer las estrategias implementadas a nivel internacional por instituciones similares. Material y métodos: Se aplicó una encuesta de satisfacción entre los médicos residentes de especialidades quirúrgicas en nuestra institución, ésta fue respondida de manera anónima. Resultados: Nuestros resultados evidencian una satisfacción de $66 \%$ con la nueva modalidad educativa en línea y un aumento en las horas semanales de estudio reportadas. Sin embargo, persisten las deficiencias en la percepción de la utilidad clínica de los contenidos, exposición quirúrgica y percepción del estado emocional. Conclusiones: El cambio a una modalidad educativa en línea se ha aceptado de manera parcial; no obstante, los médicos examinados han reportado deficiencias en el mismo y disminución de la exposición quirúrgica.

Palabras clave: Educación médica, cirugía ortopédica, COVID-19, satisfacción laboral.

Nivel de evidencia: IV

\section{ABSTRACT}

Introduction: The COVID-19 pandemic required a drastic restructuring of health care services and personnel. Medical postgraduate education programs are affected, giving priority to the care of patients infected with COVID-19. Objective: To measure the satisfaction of surgical specialty residents during the restructuring of educational curriculum during residency training and summarize, as well, the changes made by international institutions with education programs like ours. Materials and methods: We designed a satisfaction survey, taken anonymously by the residents in our institution. Results: Results show that $66 \%$ of the residents accepted the changes as satisfactory. The time dedicated to individual study also increased. Nonetheless, most residents feel a decrease in quality educational content and exposure to surgical procedures. Conclusions: During the COVID-19 pandemic the online models are useful to continue medical education, but certain areas such as operating theatre exposure are still lacking.

Keywords: Medical education, orthopaedic surgery, COVID-19, job satisfaction.

Level of evidence: $I V$
${ }^{*}$ Residente de cuarto año, Ortopedia y Traumatología. Centro Médico ABC. Ciudad de México.

Recibido: 02/03/2021. Aceptado: 30/06/2021.
Correspondencia:

Jorge Luis Martínez-Peniche

E-mail: jorchmartinez@gmail.com 


\section{INTRODUCCIÓN}

La declaratoria de pandemia de COVID-19 por el director general de la Organización Mundial de la Salud ${ }^{1}$ alertó a los sistemas de salud globalmente sobre la necesidad de la rápida reasignación de recursos humanos y materiales para la atención de la enfermedad emergente. Dicha pandemia ha afectado la práctica de casi todas las especialidades médicas que se han reestructurado para atender de manera oportuna el problema. ${ }^{2}$

El uso de recursos humanos para la atención durante la pandemia incluye necesariamente a médicos en formación en pregrado y postgrado, los cuales han respondido a las necesidades emergentes que implica el COVID-19. Estos cambios afectan directamente a la educación de los médicos residentes, por lo que se han implementado medidas que permitan reestructurar los modelos educativos y minimizar el impacto de la pandemia sobre éstos.

Desde la perspectiva de la educación médica, se identificaron problemas relacionados con la instrucción de médicos residentes en nuestro hospital, como la necesidad de apoyo a servicios de Urgencias, cancelación de eventos masivos como congresos, reuniones y conferencias, así como clases presenciales en la modalidad tutor-alumnos.

Este artículo tiene como objetivo describir las adaptaciones realizadas durante la pandemia y en los meses posteriores para minimizar el impacto de la drástica reducción de oportunidades en la educación. De igual modo, medimos la satisfacción de los residentes de especialidades quirúrgicas en nuestra institución (Cirugía General, Ginecología y Obstetricia, y Ortopedia y Traumatología) con el nuevo modelo de enseñanza con una encuesta aplicada de manera virtual.

\section{Modelo educativo actual} en el Centro Médico ABC

Los postgrados médicos del Centro Médico ABC son avalados por la Facultad de Medicina de la Universidad Nacional Autónoma de México (UNAM). Al inicio de la década de los 90 se diseñó el Plan Único de Especialidades Médicas (PUEM), que tiene como finalidad homogeneizar la formación académica y profesional del especialista en diversas sedes hospitalarias mediante un currículum en común acorde a la especialidad. ${ }^{3}$ El Plan Único fundamenta su plan académico en tres bloques:

1. Prestación de atención médica.

2. Desarrollo de la investigación.

3. Labor educativa.
Las especialidades quirúrgicas en el Centro Médico $\mathrm{ABC}$ realizan labor asistencial y de enseñanza, de manera general, bajo supervisión de un médico especialista tratante y profesor titular de curso. Esto logra brindar en todo momento retroalimentación en los tres bloques al especialista en entrenamiento, que consisten en:

1. Atención médica: consulta, atención en sala de urgencias, examen clínico, intervención quirúrgica, procedimientos diagnósticos o terapéuticos, estudio histopatológico, estudios de imagen y su respectiva interpretación, así como rehabilitación de los pacientes. Siendo la atención médica una continua aplicación de conocimientos.

2. Investigación clínica: se basa en la publicación de casos clínicos, revisión de casos, artículos científicos, tesis de investigación, seminario de investigación a cargo de la Dirección de Enseñanza, entre otras.

3. Función educativa: se refiere a la educación en docencia con base en el temario del PUEM de los cuatro años académicos que forman la especialización en Ortopedia y Traumatología. Los temas se distribuyen acorde a los años de especialización de los residentes de esta sede. Simulación en Ortopedia y Artroscopia con simuladores de última generación. Educación a otros servicios y servicio de enfermería en temas selectos de Ortopedia. Enseñanza a médicos internos de pregrado.

Un ejemplo del modelo educativo previo a la pandemia en nuestro hospital es el currículum de la especialidad en Ortopedia y Traumatología: clases presenciales diarias, de lunes a jueves, totalizando 160 clases al año, divididas en módulos educativos por segmento corporal (hombro, codo y antebrazo, muñeca y mano, pelvis y acetábulo, columna, cadera, rodilla, pie y tobillo). Se incluye en el programa clases de ciencias básicas y temas selectos en fracturas, artroscopia, reemplazos articulares y ortopedia pediátrica. Semanalmente se realiza una sesión de casos clínicos intervenidos en nuestra institución, en modalidad residente presentador-cirujano experto asesor. Se evalúan la capacidad integrativa, los conocimientos médicos y la aplicación clínica del conocimiento.

\section{Opciones de reestructuración}

Dentro del gremio médico, un número considerable de médicos residentes están adheridos a un currículum en el cual su preparación y evaluaciones requie- 
ren exposición clínica. La pandemia de COVID-19 requirió la cancelación del método tradicional de enseñanza médica por la necesidad de reestructuración de los sistemas de salud a nivel mundial.

Al tomar medidas de aislamiento social y cancelación de reuniones físicas y exposición del personal de salud al virus, se tuvo la necesidad de buscar opciones virtuales o no presenciales de educación a médicos en formación.

Producto de la pandemia de SARS en 2009, Lim y colaboradores ${ }^{4}$ proponen rediseñar la enseñanza médica de manera virtual y métodos de simulación en caso de nuevos brotes de enfermedades como el SARS. Se pueden extrapolar estos planes a la situación actual vivida por la pandemia de COVID-19.

En Estados Unidos se implementaron tres fases de educación médica por el Accreditation Council for Graduate Medical Education (ACGME) dependiendo del número de casos de COVID-19 y requerimientos por hospital: 5

1. Primera fase: las labores se llevan a cabo de manera normal.

2. Segunda fase: se implementa en respuesta de la demanda clínica del hospital, sabiendo que es probable la movilización de residentes a otros servicios. Permitiendo únicamente clases en línea y revisión de casos y artículos de manera virtual.

3. Tercera fase: se considera estado de emergencia por pandemia y se suspende toda enseñanza y evaluaciones por 30 días, permitiendo a los residentes enfocarse a labores asistenciales emergentes.

En el caso específico de la educación en Ortopedia, el American Board of Orthopaedic Surgery respondió incrementando el tiempo fuera de las actividades clínicas asistenciales cuatro a seis semanas por año, dando más flexibilidad y tiempo de aprendizaje en médicos residentes de Ortopedia. Programas de entrenamiento de alta especialidad (fellowships) avalados por la American Association of Hip and Knee Surgeons decidieron iniciar sesiones didácticas de manera virtual mediante el programa FOCAL $(\mathrm{Fe}$ llows Online COVID-19 AAHKS Learning). ${ }^{5}$ Este programa consiste en sesiones virtuales y diplomados con peso curricular que coincidan en zonas horarias a la mayor cantidad de médicos en formación.

\section{Modelo adoptado en nuestra institución}

En atención a guías internacionales y recomendaciones emitidas por organismos como la Organización Mundial de la Salud, ${ }^{1}$ la Academia Americana de
Cirugía Ortopédica ${ }^{6}$ y el Colegio Americano de Cirujanos, ${ }^{7}$ nuestra institución implementó cambios en la práctica diaria de las especialidades quirúrgicas. En el caso específico de Ortopedia y Traumatología, atendiendo las recomendaciones de la American Academy of Orthopaedic Surgeons (AAOS), se realizaron los siguientes cambios:

1. Cancelación de todos los procedimientos ortopédicos considerados electivos.

2. Autorización de tratamiento a procedimientos ortopédicos urgentes descartando infección por COVID-19 mediante prueba de PCR y tomografía de tórax.

3. Cancelación de todas las juntas y clases presenciales para médicos residentes.

4. Apoyo a personal del Departamento de Urgencias por parte de médicos residentes.

5. Reasignación de un campus específico para la atención de pacientes COVID-19 y aceptación de pacientes referidos de centros médicos del sistema público.

Con la intención de disminuir el contacto con el entorno hospitalario de los residentes, se iniciaron labores mediante trabajo en turnos de 24 horas entre todos los residentes disponibles. Un residente de primer año a cargo de los pacientes hospitalizados en piso y valoración inicial de urgencias y un residente de mayor jerarquía encargado de complementar la valoración de urgencias y de asistir en las cirugías requeridas durante el turno.

Se tomó la decisión de cambiar la estructura de las clases hacia la modalidad en línea. Todas las clases se toman con la plataforma de videoconferencias Zoom (Zoom Video Communications, Inc. San Jose, California. EE.UU.), Google Hangouts (Google LLC, Mountain View, California. EE.UU) o similares, bajo la modalidad residente expositor-experto asesor. Asimismo, se implementaron nuevas modalidades y dinámicas educativas, complementando la formación de los residentes. Las nuevas modalidades educativas se resumen en la Tabla 1.

\section{MATERIAL Y MÉTODOS}

Se diseñó una encuesta de satisfacción educativa para los residentes que conforman las especialidades quirúrgicas del Centro Médico ABC.

Se consultó el protocolo y la encuesta a los comités de enseñanza e investigación, y se obtuvo su aprobación y registro con la clave ABC-21-12. 
Tabla 1: Nuevas modalidades educativas adoptadas.

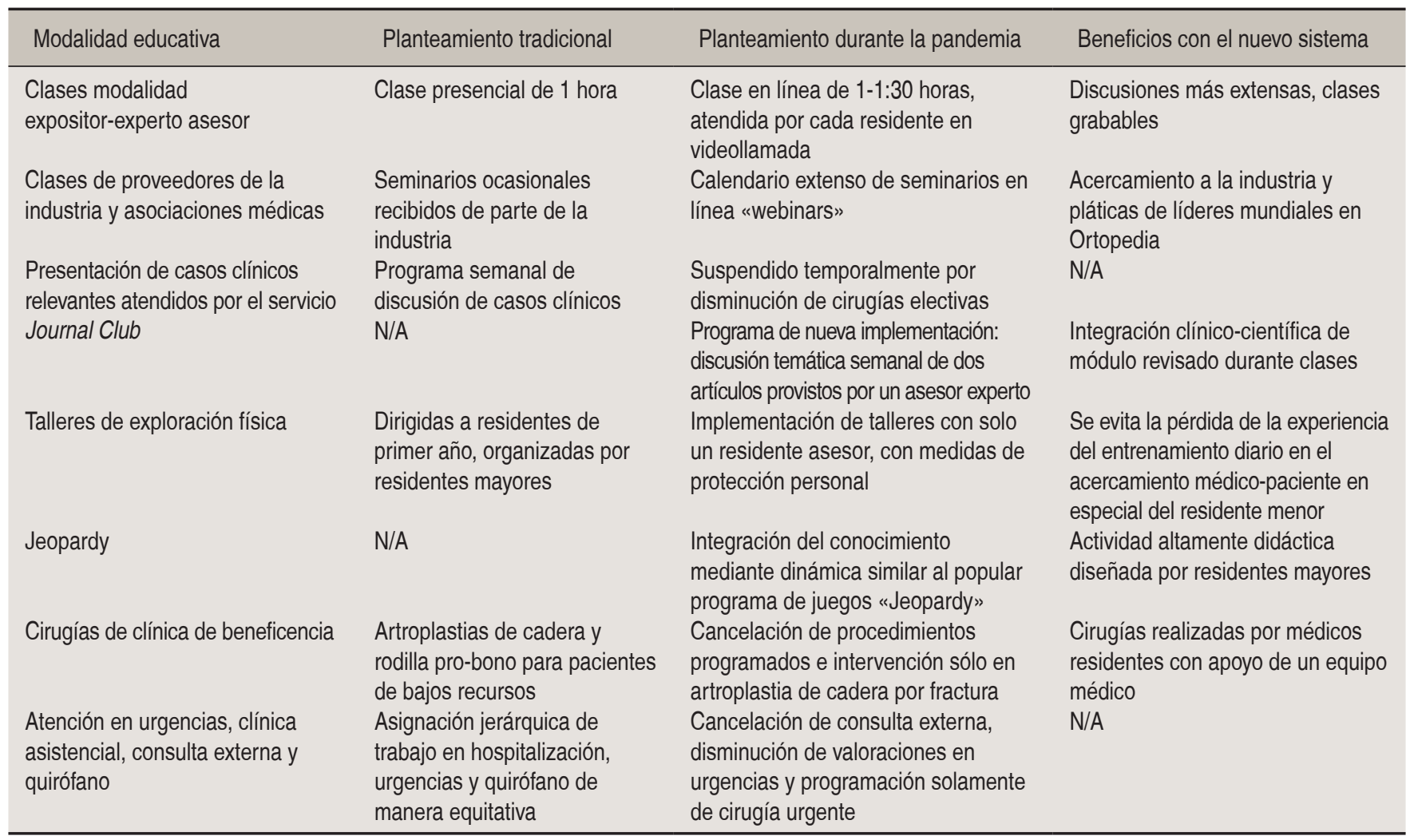

El instrumento diseñado mide la satisfacción del médico residente en diferentes ámbitos educativos desde el inicio de la pandemia de COVID-19. Se evaluaron los siguientes parámetros: evaluación de contenido, nivel educativo y utilidad práctica de los currículos virtuales, horas semanales de estudio individual previo y posterior a la pandemia, exposición quirúrgica y una pregunta de cribaje de estado emocional.

Se extendió la invitación a todos los médicos residentes quirúrgicos de segundo, tercer y cuarto año a través de los jefes de residentes de cada especialidad a participar. Previa obtención de consentimiento informado, se obtuvieron 12 respuestas anónimas en la encuesta.

\section{RESULTADOS}

Se obtuvo la participación de tres residentes de Ortopedia, cinco de Cirugía General y cuatro de Ginecología. Los resultados obtenidos evidencian una satisfacción general con el nuevo modelo educativo (66\%). Se reportó una transición adecuada (respuestas «Satisfactoria» $\mathrm{y}$ «Excelente») al nuevo modelo educativo en $66 \%$ de los casos. De igual modo, el nivel educacional se reportó como «Satisfactorio» y/o «Excelente» en $83 \%$ de los casos.

La satisfacción en cuanto a utilidad clínica de los contenidos fue de $50 \%$ y de manera casi universal (92\%) los residentes disminuyeron o mantuvieron su mismo nivel de exposición quirúrgica. Sólo $25 \%$ de los residentes reportaron que su aprendizaje ha aumentado con la modalidad en línea de los contenidos curriculares.

Las horas de estudio reportadas aumentaron significativamente, con reportes de 15 o más horas de estudio individual semanal en cuatro de los entrevistados, comparado con el periodo previo a la pandemia, cuando sólo dos acumulaban esa cantidad. Estos resultados se resumen en las Figuras 1 y 2.

Por último, se reportó un estado emocional «Regular» o «Deficiente» en 66\% de los entrevistados durante el transcurso de la pandemia.

\section{DISCUSIÓN}

Se decidió aplicar este cuestionario solamente a residentes de segundo a cuarto año de especialidad du- 
rante el año 2021, debido a que ellos experimentaron la transición del modelo educativo presencial tradicional al modelo virtual.

De acuerdo con los resultados de nuestra encuesta podemos obtener conclusiones importantes. La disminución en el trabajo hospitalario habitual durante 2020 generó que incrementara el tiempo utilizado para el estudio de los residentes; sin embargo, sólo $33 \%$ de los entrevistados cumple con las 15 horas semanales de estudio individual recomendadas por la UNAM y establecidas en el PUEM. ${ }^{3}$

De igual manera, aunque se reportó una transición adecuada a las nuevas modalidades de enseñanza, no se tiene la percepción de que los contenidos educativos tengan relevancia clínica tan importante como cuando se abordan en clases presenciales. Es la opinión de los autores que las clases presenciales y la enseñanza en ambientes clínicos favorecen esta percepción de «utilidad clínica» del currículum.

$\mathrm{Al}$ ser voluntarios en nuestro estudio residentes de especialidades quirúrgicas, se consideró relevante el investigar su exposición a procedimientos quirúrgicos. De forma casi universal se reportó que ha disminuido el tiempo en quirófano de los residentes. Durante los primeros meses de la pandemia se suspendieron de manera abrupta todos los casos electivos, dejando sólo los procedimientos considerados urgencias absolutas y relativas como parte de la exposición quirúrgica de los médicos en formación.

El cambio repentino de las modalidades de trabajo y las cambiantes necesidades del hospital en el momento de crisis afectaron de manera medible el estado emocional de nuestros residentes. Los voluntarios reportaron en su mayoría un estado emocional no satisfactorio durante los meses de aislamiento y currículo virtual.

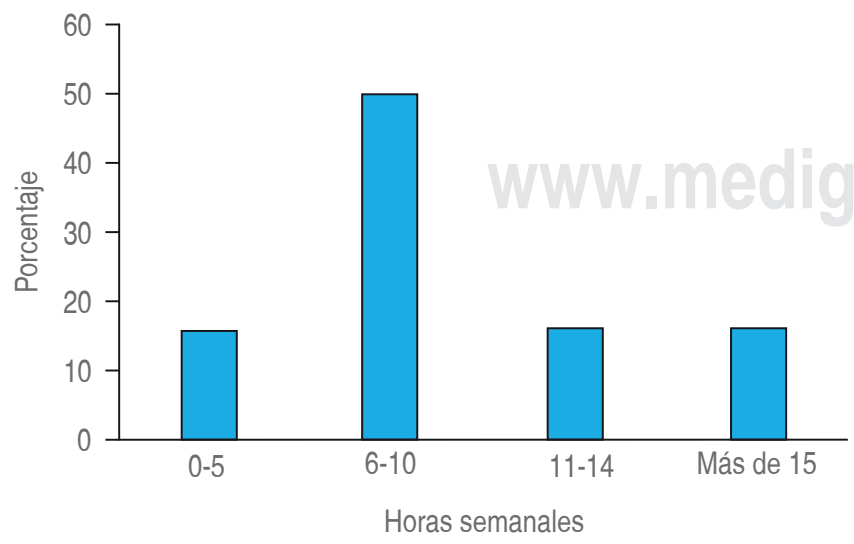

Figura 1: Horas semanales de estudio previo a la pandemia.

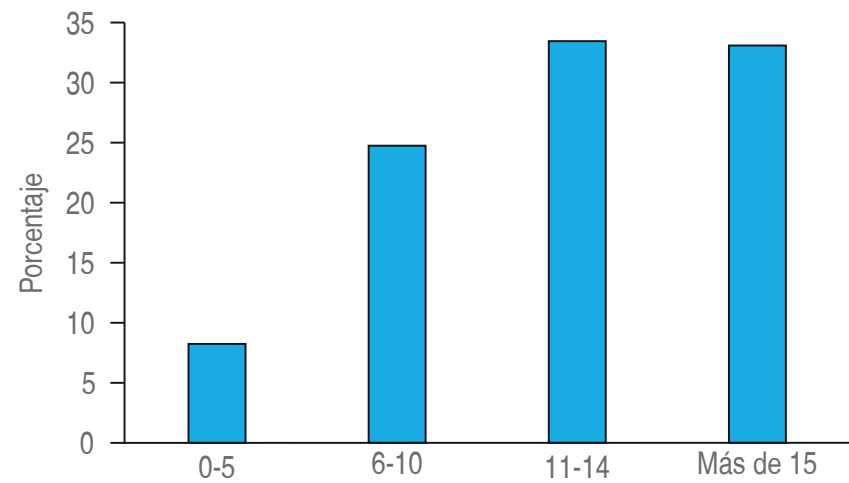

Figura 2: Horas de estudio semanal durante la pandemia.

Múltiples autores ${ }^{4,8}$ han descritos diferentes estrategias para manejar los programas de educación médica durante estos tiempos de crisis global, incluyendo la migración a un ambiente digital de enseñanza, programas de simulación con maniquíes y la limitación estricta de contacto clínico con personal médico de pregrado y estudiantes.

Schwartz y colaboradores ${ }^{9}$ han demostrado mediante su reciente artículo publicado que se puede reestructurar rápida y efectivamente el trabajo hospitalario de los residentes. La reasignación del capital humano en dos equipos (uno trabajando presencialmente y otro remotamente), intercambiando roles cada 15 días, permite una buena distribución de la carga laboral y atiende a los principios de seguridad del personal médico y pacientes. Stambough ${ }^{5}$ y su grupo describen cómo ha cambiado en su institución la educación a residentes de ortopedia y grupos de formación en alta especialidad. Mencionan a la pandemia como catalizador de cambio en las oportunidades didácticas y la implementación de nuevos métodos de enseñanza.

Hemos tomado estas últimas publicaciones como base para la reestructuración del trabajo clínico y el apoyo a diferentes servicios dentro de nuestro hospital. Sin embargo, existen todavía muchas áreas de oportunidad en este tema.

\section{CONCLUSIONES}

La pandemia actual ha orillado a todo el gremio médico a realizar una rápida reestructuración de los programas de aprendizaje en todas las áreas, y nuestros programas de residencia no son la excepción. Se aprecia el buen aprovechamiento de la nueva cantidad de tiempo disponible y consideramos que nuestras estrategias pueden ser aplicadas a otros centros 
hospitalarios. Sin embargo, existe una brecha entre los contenidos educacionales y su aplicación y entendimiento pleno. De igual manera, debemos tomar en cuenta el estado emocional de los médicos en formación y solicitar el apoyo de profesionales de la salud mental para mejorar este parámetro.

Consideramos tener la tecnología de nuestro lado y debemos emplearla al máximo. Es inevitable el cambio que realizó esta pandemia y tenemos que estar anticipando una nueva normalidad. Los hechos nos indican que muchas modificaciones en los programas educativos serán necesarias y se deberán ir perfeccionando con el tiempo.

\section{AGRADECIMIENTOS}

Agradecimiento especial a la Dra. Sahara Hurtado y a la Dra. Regina Ruiz por su colaboración en la elaboración de este documento.

\section{REFERENCIAS}

1. Organización Mundial de la Salud. WHO Director-General's opening remarks at the media briefing on COVID-19 - 11 March 2020. [Obtenido el día 20/05/20] Disponible en: https:// www.who.int/dg/speeches/detail/who-director-general-sopening-remarks-at-the-media-briefing-on-covid-19---11march-2020
2. Wang C, Horby PW, Hayden FG, Gao GF. A novel coronavirus outbreak of global health concern. Lancet. 2020; 395 (10223): 470-473.

3. Facultad de Medicina UNAM. Plan Único de Especializaciones Médicas en Ortopedia. [Recuperado el 20/04/2020] Disponible en: http://www.fmposgrado.unam.mx/especialidades/que-est.html

4. Lim EC, Oh VM, Koh DR, Seet RC. The challenges of "continuing medical education" in a pandemic era. Ann Acad Med Singap. 2009; 38 (8): 724-726.

5. Stambough JB, Curtin BM, Gililland JM, Guild GN 3rd, Kain MS, Karas V et al. The past, present, and future of orthopedic education: lessons learned from the COVID-19 pandemic. J Arthroplasty. 2020; 35 (7S): S60-S64.

6. American Academy of Orthopedic Surgeons. Navigating the COVID-19 Pandemic. Comunicado. [Recuperado el día 24/04/2020] Disponible en: https://www.aaos.org/globalassets/ about/covid-19/aaos-clinical-considerations-during-covid-19.pdf

7. American College of Surgeons. COVID-19: recommendations for management of elective surgical procedures. Comunicado. [Recuperado el día 24/04/2020] Disponible en: https://www. facs.org/covid-19/clinical-guidance/elective-surgery

8. Ahmed H, Allaf M, Elghazaly H. COVID-19 and medical education. Lancet Infect Dis. 2020; 20 (7): 777-778.

9. Schwartz AM, Wilson JM, Boden SD, Moore TJ Jr, Bradbury TL Jr, Fletcher ND. Managing resident workforce and education during the COVID-19 pandemic: evolving strategies and lessons learned. JB JS Open Access. 2020; 5 (2): e0045.

Declaración de conflicto de intereses: Ninguno de los autores reporta conflicto de intereses.

Financiamiento: No se recibió ningún financiamiento para la realización de este artículo. 